\title{
An optimal design of driving mechanism in a 1 degree of freedom (d.o.f.) anthropomorphic finger
}

\author{
M. Ceccarelli ${ }^{1}$, N. E. N. Rodríguez ${ }^{1}$, G. Carbone ${ }^{1}$ \\ and C. Lopez-Cajùn ${ }^{2}$ \\ ${ }^{1}$ LARM: Laboratorio di Robotica e Meccatronica, DiMSAT-Università di Cassino, Via Di Biasio 43, \\ 03043 Cassino (Fr), Italy \\ ${ }^{2}$ Facultad de Ingenieria, Universidad Autonoma de Queretaro, Cerro de las Campanas $s / n$, \\ 76010 Santiago de Queretaro, Queretaro, Mexico
}

\begin{abstract}
Mechanisms can be used in finger design to obtain suitable actuation systems and to give stiff robust behavior in grasping tasks. The design of driving mechanisms for fingers has been attached at LARM in Cassino with the aim to obtain one degree of freedom actuation for an anthropomorphic finger. The dimensional design of a finger-driving mechanism has been formulated as a multi-objective optimization problem by using evaluation criteria for fundamental characteristics regarding with finger motion, grasping equilibrium and force transmission. The feasibility of the herein proposed optimum design procedure for a finger-driving mechanism has been tested by numerical examples that have been also used to enhance a prototype previously built at LARM in Cassino.
\end{abstract}

Key words: Grasping devices, anthropomorphic fingers, driving mechanisms, optimal design.

\section{INTRODUCTION}

The design of anthropomorphic fingers still attracts great interest for applications in universal grasping devices, biomedical prosthesis, and investigations on multiple contact grasps.

Researchers have investigated different types of finger devices for achieving the grasping and handling of objects. Several successful designs for anthropomorphic fingers have been developed in the past, like those that are used for example in Stanford/JPL Hand (Mason and Salisbury 1985), DLR Hand (Butterfass et al 2001), BUAA Hand (Zhang et al 2001), Colobi Hand (Gosselin et al 1993), Barrett Hand (Townsend 2000), TUAT/Karlsruhe Hand (Fukaya et al 2000), Turin Hand (Raparelli et al 2000), and TBM Hand (Dechev et al 1999). Most of the available multi-fingered prototypes have a high number of degrees of freedom, a complex control, and a high cost.

Most of those hands are still used for enhanced applications, laboratory tests or researches. But even in the

\footnotetext{
Corresponding Author:

M. Ceccarelli

LARM: Laboratorio di Robotica e Meccatronica

DiMSAT_Università di Cassino

Via Di Biasio 43, 03043 Cassino (Fr), Italy

Email: ceccarelli@unicas.it
}

market hands are available for industrial and nonindustrial applications, for example, Barrett Hand (Barrett Hand), and among the above-mentioned ones, the Stanford/JPL Hand (Stanford/JPL Hand) is one of the most successful.

The above-mentioned hands are quite complicated in the mechanical design since the several parts and they show problems in actuation economy in terms of control simplicity, weight, mechanical sizes of transmissions, and operation programming.

Since the early 1990 s at LARM, a research line has been devoted to a project for designing a low-cost easyoperation hand. The mechanics of human hand grasping has been mimicked in robotic devices by experiencing their feasibility through prototypes and specific tests that are reported in Ceccarelli (1997), Deibe et al (1997), Figliolini and Ceccarelli (1999, 2000, 2002), Carbone et al (2002), Penisi et al (2002), Figliolini et al (2003), Jauregui et al (2003), Ceccarelli et al (2003). Recently, at LARM, successful prototypes of three-fingered hands have been built and tested with satisfactory results as reported in Jauregui et al (2003), Ceccarelli et al (2003), Nava et al (2004a, 2004b).

The fundamental characteristics of the LARM designs for anthropomorphic fingers can be recognized in two aspects, namely having 1 degree of freedom (d.o.f.) driving mechanisms for reproducing a human grasping of regular objects, and a mechanical design with compact, 


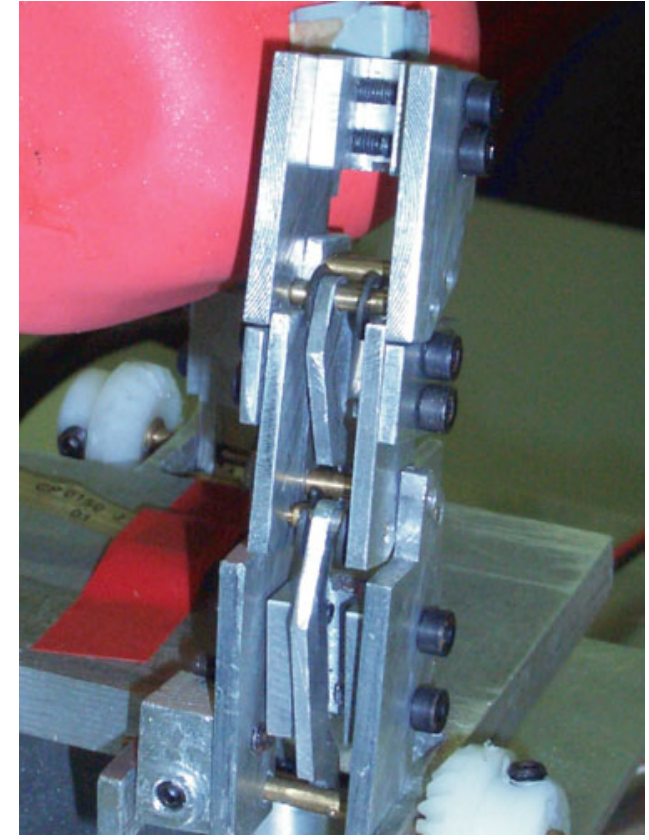

Figure 1 The new 1 d.o.f. finger prototype designed at LARM in Cassino.

stiff, and robust solution. At LARM a prototype of an anthropomorphic finger with 1 d.o.f. has been built with human size after some considerations for optimal solution in its mechanical design, as shown in Figure 1. However, an open problem can be still advised in a design formulation that can fulfill design requirements within a rational procedure.

In this paper, we have reported the attempt of such a rational design procedure for optimum sizing of fingerdriving mechanisms by using fundamental characteristics regarding basically with the human-like behavior, grasp efficiency, and force transmission. Satisfactory results are reported in this paper also through numerical examples.

\section{MECHANICAL DESIGN OF A BUILT PROTOTYPE}

The investigation activity on anthropomorphic fingers at LARM has given several prototypes, as reported in Figliolini and Ceccarelli (1999, 2000, 2002), Carbone et al (2002), Penisi et al (2002), Figliolini et al (2003), Jauregui et al (2003), Ceccarelli et al (2003a, 2003b), Nava et al (2004b).

In Figure 1 a last design of a finger with human size is shown by emphasizing on the compactness aspects. In order to have a low-cost lightweight prototype, it has been decided to manufacture it by using commercial aluminum alloy. The small dimensions of the links require a careful mechanical design and manufacturing of each component. Therefore, it has been preliminarily developed a 3D CAD model of the prototype in order to check also the assembly feasibility of all the components, as illustrated in Jauregui et al (2003), Ceccarelli et al (2003a, 2003b), Nava et al (2004b). Moreover, the developed 3D model has been used

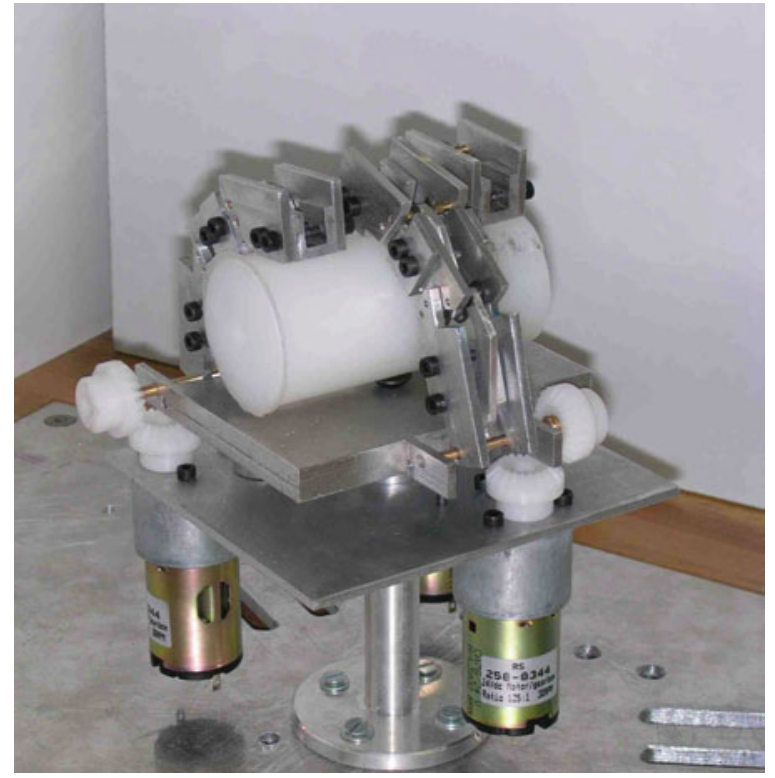

Figure 2 A prototype of three-fingered LARM hand with 1 d.o.f. anthropomorphic fingers of human sizes.

to carry out several simulations of the operation of the finger. These simulations have been useful in order to check the feasibility of the proposed dimensions for avoiding link interference and for properly mimicking the human index finger.

Figure 2 shows the application of the 1 d.o.f. anthropomorphic finger of Figure 1 in a three-fingered hand. Even the three-fingered hand design has been tested previously by using simulations with 3D CAD suitable programming (Jauregui et al 2003, Ceccarelli et al 2003b). Those simulations have been useful also to properly select the installation of low-cost DC motors and to check the efficiency of several palm designs. Figure 2 shows the solution that has been designed for a suitable application on a PUMA562 robot that is available at LARM. In particular, the DC motors have been selected for an easy-operation of the system through a low-cost PLC.

In Figure 3 the driving mechanism of the anthropomorphic finger is sketched from kinematics viewpoint together with the design parameters. The driving mechanism is a series of crossed-four bar linkages whose proportions are designed for the prototype to reproduce the human motion of grasping.

The kinematic architecture of the scheme of Figure 3 permits to mimick the 3 d.o.f. movement of human fingers through a 1 d.o.f. mechanism movement. Referring to Figure 3, it can be observed that any movement of the first phalanx through links $l_{31}, l_{12}, l_{51}$ will actuate the second phalanx through links $l_{41}, l_{52}, l_{32}$. Similarly, any motion of the second phalanx will actuate the third phalanx through links $l_{42}, l_{53}$. This solution has been chosen since it also provides a suitable stiffness of the system at a grasp configuration. In addition, because of the linkage design, the finger-driving mechanism can be robust, low-cost, and it 


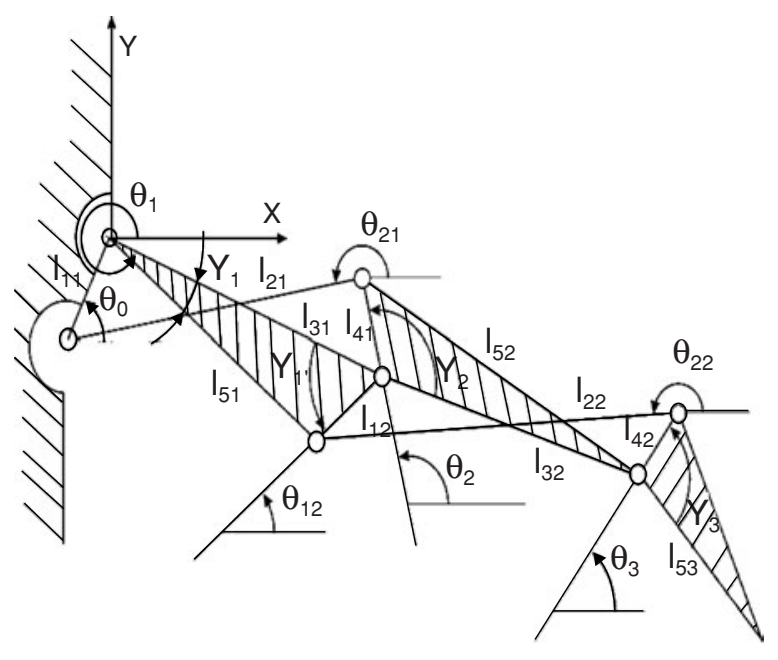

Figure 3 The kinematic diagram of the 1 d.o.f. anthropomorphic finger of Figures 1 and 2.

can be conveniently embedded in the finger body with the aim to obtain a human-size artificial finger. In addition, special care has been addressed to the dimensional synthesis of the articulated mechanisms in order to obtain the needed transmission ratios, to avoid link interference, and to achieve suitable mobility. This preliminary design has been studied and obtained with satisfactory results by looking at the different aspects separately, and by achieving a dimensional synthesis of the mechanism by means of traditional techniques.

\section{PROBLEMS FOR 1 D.O.F. ANTHROPOMORPHIC FINGER MECHANISM}

The design of an anthropomorphic finger must fulfill basic requirements on:

- human-like motion and grasp;

- compact size; and

- actuation lightweight and efficiency.

The above-mentioned aspects can be evaluated numerically for analysis and design purposes.

In particular, human-like motion can be described by looking at the deviation of the motion characteristics between a human test and operation of a designed finger mechanism. The kinematics of the motion of human fingers has been analyzed by using video recording and photo sequence, and the trajectory can be represented as a path function of the articulation joints. Figure 4 shows a photo sequence of the human finger motion in approaching the grasp of a cylinder.

A numerical evaluation of the human-like motion that can be reproduced in an anthropomorphic finger mechanism can be obtained by looking at the joint motion as referring to a finger design scheme. Referring to the kinematic design of Figure 3, the experimental data in Figure 5 can be used to identify an interpolating cubic line for the phalanx angle joints $\theta_{2}$ and $\theta_{3}$ as functions of $\theta_{1}$
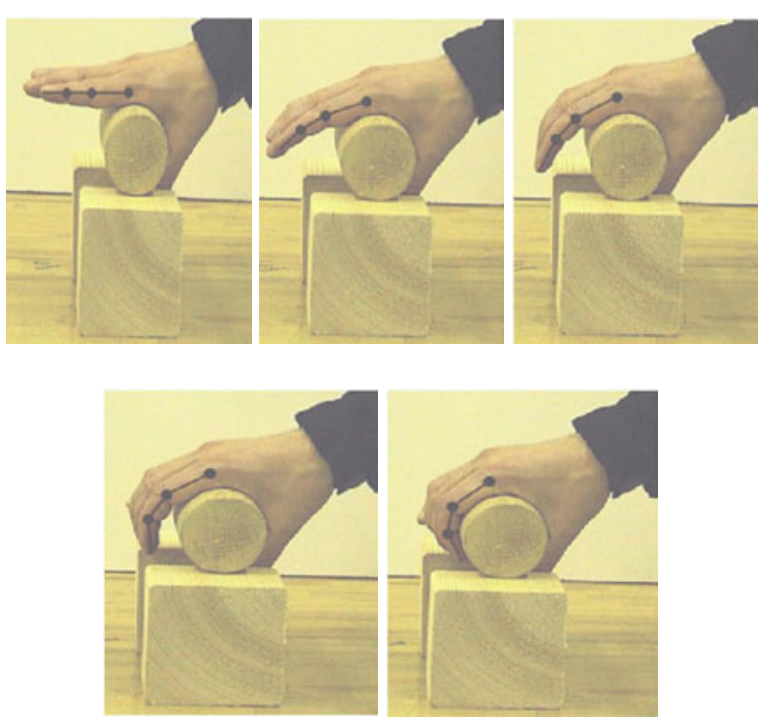

Figure 4 Photo sequence of a test of human grasping at LARM.

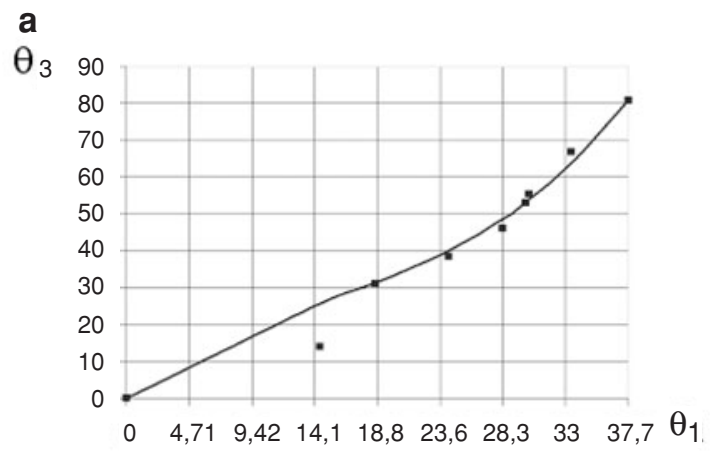

b

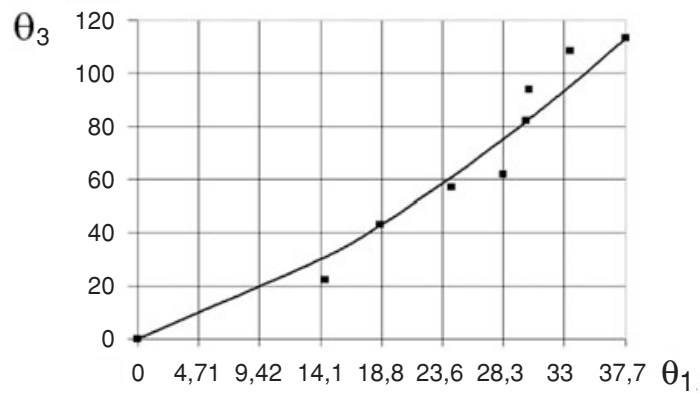

Figure 5 Numerical evaluation of the test in Figure 4 in the form of experimental measures as square cubic dots and as cubic line interpolation by referring to the scheme of Figure 3 : (a) $\theta_{2}$ vs. $\theta_{1}$; (b) $\theta_{3}$ vs. $\theta_{1}$.

angle joint of first phalanx that is the input 1 d.o.f. motion. Thus, an interpolating cubic line can be written as

$$
h_{\theta_{i}}=c_{1 \mathrm{~h} i} \theta_{1 \mathrm{~h}}+c_{2 \mathrm{~h} i} \theta_{1 \mathrm{~h}}^{2}+c_{3 \mathrm{~h} i} \theta_{1 \mathrm{~h}}^{3}
$$

in which the coefficients $c_{\text {jhi }}(j=1,2,3 ; i=2,3)$ are determined by using the experimental data. For the case of Figure 4, the numerical evaluation is shown in Figure 5 as the continuous lines that are obtained from experimental measures. 


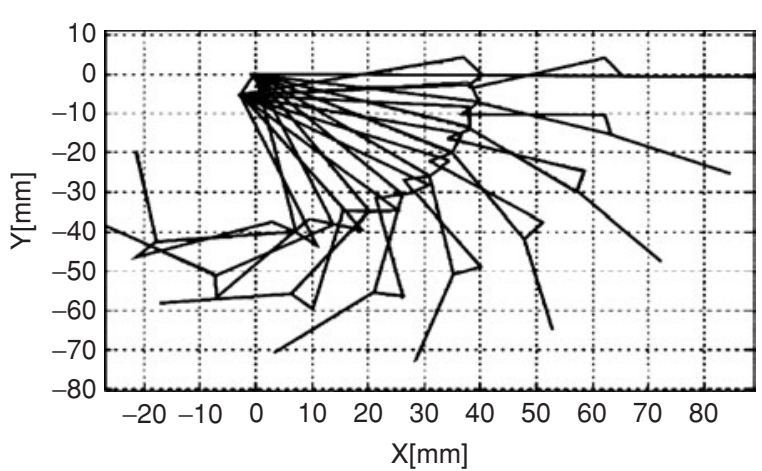

Figure 6 A simulation of the finger-driving mechanism of Table 1 and Figure 3 mimicking the human motion of Figure 4.

Table 1 Sizes of the finger prototype of Figure 1 with kinematic design of Figure 3

\begin{tabular}{llllll}
\hline$i$ & $l_{1 i}(\mathrm{~mm})$ & $l_{2 i}(\mathrm{~mm})$ & $l_{3 i}(\mathrm{~mm})$ & $l_{4 i}(\mathrm{~mm})$ & $l_{5 i}(\mathrm{~mm})$ \\
\hline 1 & 8.5 & 40.9 & 40.5 & 5.4 & 43.7 \\
2 & 4.8 & 26.0 & 25.0 & 5.4 & 22.0 \\
\hline
\end{tabular}

In the example for design purposes, nine different configurations have been measured experimentally, as reported in Figure 5(a) and (b). The continuous line is a cubic interpolation of the experimental data that are reported as square black dots.

A numerical simulation of the designed driving mechanism of Figure 3 with sizes in Table 1 can give a finger motion as reproducing the human motion to the grasping.

With the aim to obtain the functions of equation (1), the mechanism has been sized as reported in Table 1 to give the motion of the phalanxes by using a similar cubic function in the form:

$$
f_{\theta_{i}}=c_{1 \mathrm{f} i} \theta_{1 \mathrm{f}}+c_{2 \mathrm{f} i} \theta_{1 \mathrm{f}}^{2}+c_{3 \mathrm{f} i} \theta_{1 \mathrm{f}}^{3} .
$$

The coefficients $c_{j \mathrm{f} i}(j=1,2,3 ; i=2,3)$ for fingerdriving mechanism can be determined as function of the link parameters.

A numerical simulation of the designed driving mechanism can be used to check the feasibility of the synthesized solution.

Figures 6 and 7 report satisfactory results of the simulation, mainly if compared with the plots of Figure 5 . Therefore, a criterion for achieving an optimum solution can be formulated as the difference of those finger motion functions, that is

$$
\Delta f_{i}=h_{\theta_{i}}-f_{\theta_{i}} \quad(i=2,3)
$$

where $h_{\theta_{i}}$ refers to the joint function of $\theta_{i}$ in human finger and $f_{\theta_{i}}$ is the corresponding function in the finger mechanism.

The human-like grasp can be obtained by the finger mechanism by looking at the contacts, in terms of their number and locations, and even in terms of the contact forces that can ensure a static equilibrium of the grasped
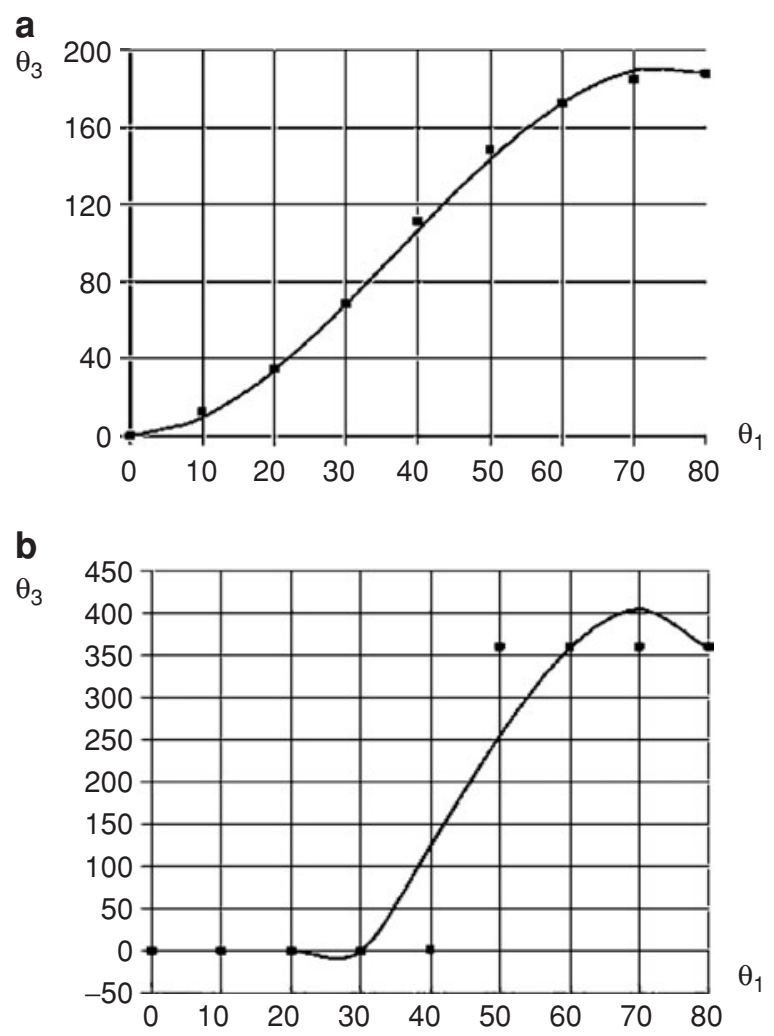

Figure 7 Results of the simulation in Figure 6 by means of closure equations as square cubic dots and as a cubic line interpolation: (a) $\theta_{2}$ vs. $\theta_{1}$; (b) $\theta_{3}$ vs. $\theta_{1}$ (angles are expressed in degrees).

object among the fingers. These aspects can be studied through suitable models and using them for design purposes.

Figure 8 shows results of a carried analysis. Referring to the grasping configuration of Figure $8(a)$, one contact for each phalanx has been assumed in the design model of Figure 8(b). The contact forces are considered in Figure 8(c) neglecting the friction components in order to evaluate the grasping efficiency mainly due to the mechanical design of the finger.

The contact aspect of the grasp is strongly related to the actuation power, and the static equilibrium is also ensured by the hyperstatic configuration of the force constraints of the grasped object between the fingers. Basic computations have been modeled by referring to the two-finger grasp of Figure 8(c).

The compact size of a human finger can be obtained in a mechanical design of an anthropomorphic finger mechanism by imposing size constraints within the design procedure.

The size $L_{\mathrm{h}}$ of human index finger as design reference can be evaluated by looking through Figures 9 and 10 at the size of the phalanxes in the form:

$$
L_{\mathrm{h}}=L_{\text {phal } 1 \mathrm{~h}}+L_{\text {phal } 2 \mathrm{~h}}+L_{\text {phal } 3 \mathrm{~h}}
$$



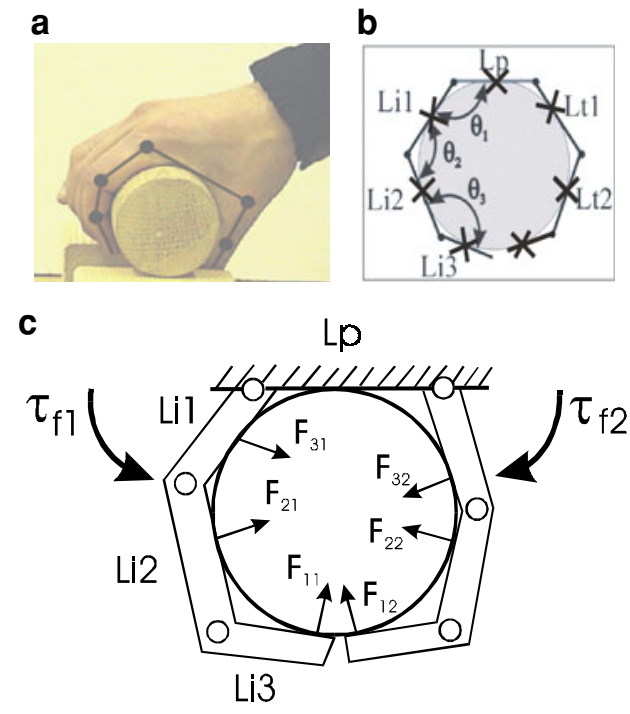

Figure 8 A scheme for human-like grasp: (a) the reference grasp test; (b) location of contacts; and (c) a model for grasping forces.

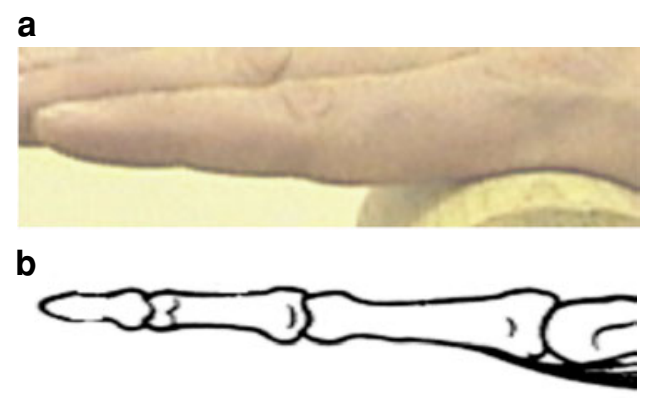

Figure 9 A human index finger as design reference for a finger-driving mechanism: (a) a photo of the reference finger; and (b) index finger skeleton (Taylor and Schwartz 1955).

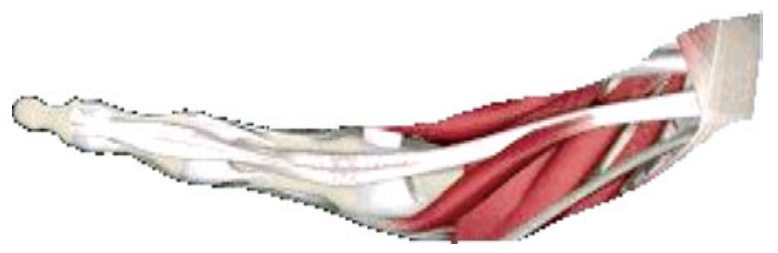

Figure 10 Muscle structure of a human index finger (Tecnoedu Homepage).

when average dimensions are considered, as reported in Carbone et al (2002), Jauregui et al (2003), Ceccarelli et al (2003a, 2003b). The size $L_{\mathrm{f}}$ of a finger mechanism can be evaluated in a similar form as

$$
L_{\mathrm{f}}=L_{\text {phal 1f }}+L_{\text {phal } 2 \mathrm{f}}+L_{\text {phal } 3 \mathrm{f}}
$$

that takes the specific expression:

$$
L_{\mathrm{f}}=\sqrt{\left(l_{21}\right)^{2}+\left(l_{41}\right)^{2}}+\sqrt{\left(l_{22}\right)^{2}+\left(l_{42}\right)^{2}}+l_{53}
$$

when refers to the link parameters of the kinematic design of Figure 3.
An optimality criterion can be formulated by referring to the difference:

$$
\Delta L=L_{\mathrm{h}}-L_{\mathrm{f}}
$$

Similarly, the problems for small size of transmission can be attached by expressing design constraints.

The actuation lightweight and efficiency is a fundamental characteristic of the nature design of human fingers. The structure of bones and muscles is quite complex in finger nature, but it is very compact and highly efficient, as shown in Figure 9.

The static equilibrium and consequent requirement of actuation power can be evaluated by referring to the models of Figure 8(c) in order to deduce fairly simple measures of the contact forces that can be monitored in experimental tests of human grasp and in fingers prototype operation. A numerical evaluation of grasping power $W_{\mathrm{f} j}$ can be formulated for the $j$ th d.o.f. driving finger mechanism of Figure 3 by looking at the actuating torque $\tau_{\mathrm{f} j}$, which is needed to exert the three contact forces $F_{i j}$ on the grasped object in Figure 8 (c) $(j=1,2 ; i=1,2,3)$. The grasping power $W_{\mathrm{f} j}$ of a grasping finger mechanism can be computed in the form:

$$
W_{\mathrm{f} j}=\tau_{\mathrm{f} j} \dot{\theta}_{1 j}=\sum_{i=1}^{3} F_{i j} V_{i j}
$$

in which $V_{i j}$ is the velocity of the contact point is in the direction of $F_{i j}$. The actuator power $W_{\mathrm{h}}$ for human finger can be computed as

$$
W_{\mathrm{h}}=\sum_{i=1}^{n} \tau_{\mathrm{h} i} \dot{\theta}_{\mathrm{h} i}
$$

in which $\tau_{\mathrm{h} i}$ is the torque and $\dot{\theta}_{\mathrm{h} i}$ is the corresponding velocity of the $i$ th articulation of the reference human index finger.

Since velocity of the grasping motion is difficult to measure and to evaluate, an optimality criterion can be formulated more conveniently by referring to the difference of the actuating torque in the form:

$$
\Delta \tau_{j}=\sum_{i=1}^{n} \tau_{\mathrm{h} i} \dot{\theta}_{\mathrm{h} i}-\tau_{\mathrm{f} j} .
$$

Summarizing, the optimality criteria for designing 1 d.o.f. finger mechanisms can be formulated by analyzing the operation of a reference human index finger and a characteristics of a feasible mechanical design. An anthropomorphic design and operation of 1 d.o.f. finger mechanism can be attached by considering the minimization of the above-mentioned optimality criteria.

\section{A FORMULATION FOR OPTIMUM DESIGN}

Because of the above-mentioned design requirements and criteria, the design of finger-driving mechanisms can be 


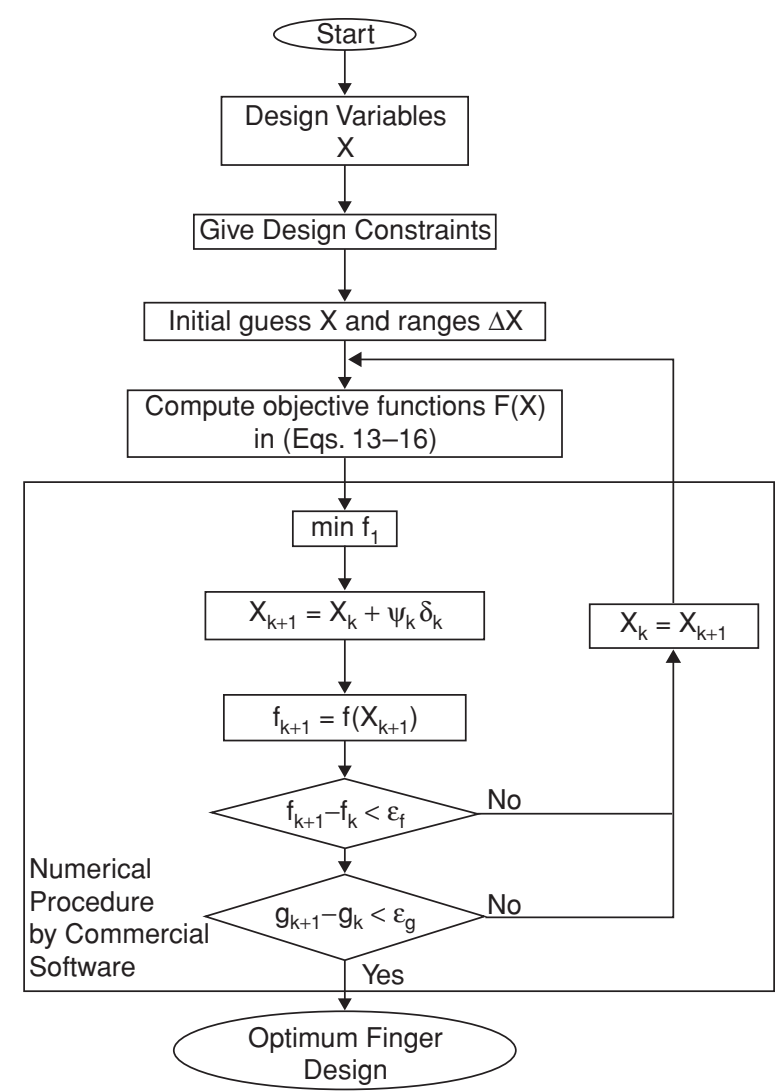

Figure 11 A numerical procedure using a commercial software package for solving the design optimization problem for finger-driving mechanisms.

conveniently attached by considering them in a simultaneous way. An optimum design procedure of 1 d.o.f. finger-driving mechanism can be formulated by using the above-mentioned evaluation criteria for finger mechanism design in a multi-objective optimization problem in the form:

$$
\min \mathbf{F}(X)
$$

subject to

$$
\mathbf{G}(X) \geq 0
$$

in which $\mathbf{F}$ is a function vector whose components are the design optimality criteria, $\mathbf{X}$ is the vector of design parameters and $\mathbf{G}$ is a function vector of constraints due to the application and characteristics of the design problem.

The numerical solution of a multi-objective optimization problem can be very complex and computationally troublesome. Several techniques are available (Vanderplaats 1984), even in commercial software packages.

The solution with weighting factors can be considered suitable from engineering viewpoint; also because it permits a designer to guide and track the significance of the optimality criteria by adopting numerical values of the weighting factors in agreement with her/his engineering experience and problem peculiarities.

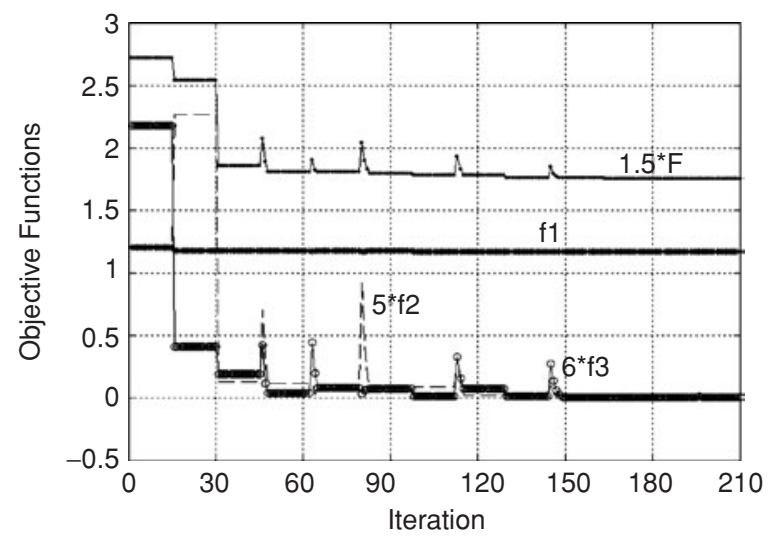

Figure 12 Evolution of the objective function and optimality criteria versus number of iterations for the numerical example.

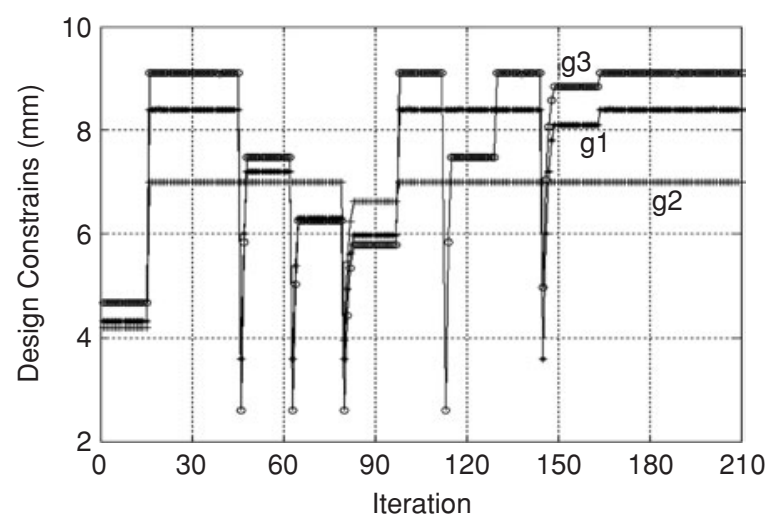

Figure 13 Evolution of the design constraints.

Therefore, optimality criteria can be used as objective functions in the form of normalized computations, and the optimum design of driving mechanisms for 1 d.o.f. anthropomorphic finger mechanism can be formulated with a weighted objective function in the form:

$$
\min \mathbf{F}(X)=w_{1} f_{1}(X)+w_{2} f_{2}(X)+w_{3} f_{3}(X)
$$

subject to

$$
\mathbf{G}(X) \geq 0
$$

with

$$
f_{1}=\sum_{i=1}^{3} \Delta f_{i}, \quad f_{2}=\frac{\Delta L}{L_{\mathrm{h}}}, \quad f_{3}=\frac{\Delta \tau}{\sum_{i=1}^{n} \tau_{\mathrm{h} i}}
$$

and

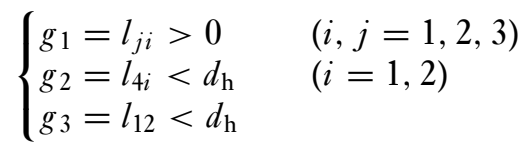

in which the normalizing values are obtained by referring to the reference human index finger. 


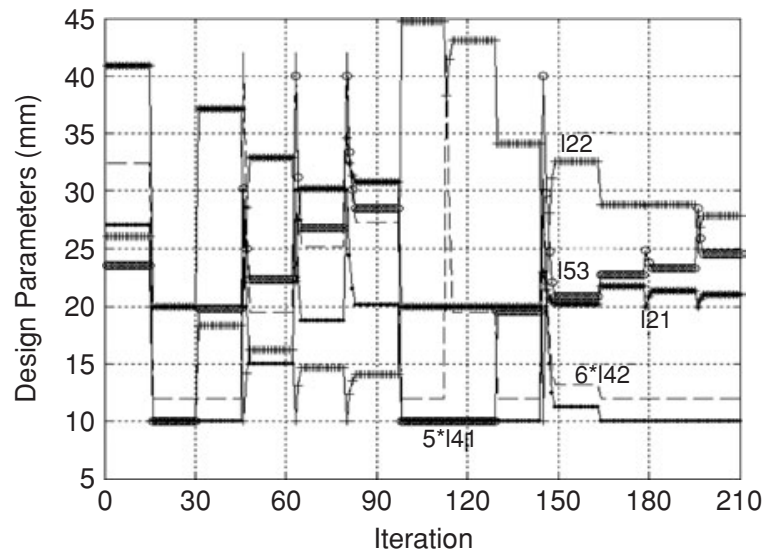

Figure 14 Evolution of design parameters $l_{22}, l_{53}, l_{21}, l_{42}, l_{41}$.

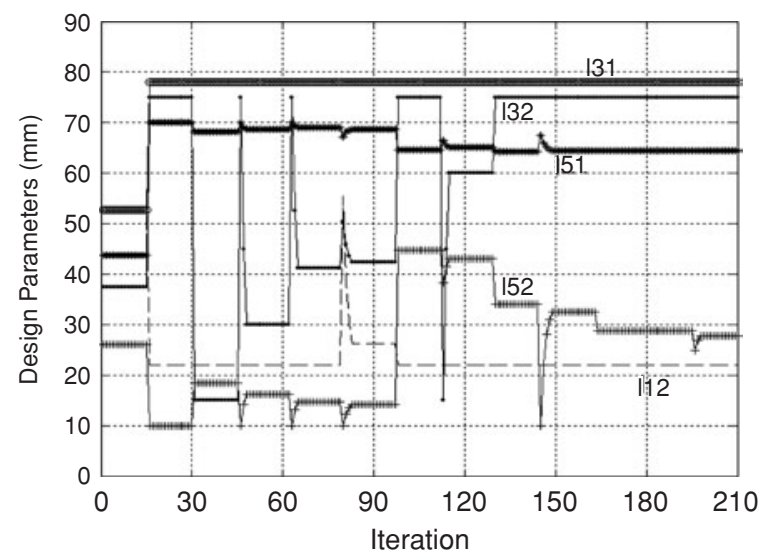

Figure 15 Evolution of design parameters $l_{31}, l_{32}, l_{51}, l_{52}, l_{12}$.

The design problem of equations (13)-(16) can be numerically solved as outlined in Figure 11 by using a numerical procedure of a commercial software package (Grace 1992), when all involved computations have been expressed for a fairly easy computer analysis.

In Figure 11 the adopted procedure for the optimal design is outlined by properly combining the formulated optimality criteria with the Sequential Quadratic Programming of the Constr algorithm in Matlab Optimization Toolbox (Grace 1992). The optimality criteria have been computed within the searching calculations for both objective functions and constraints, once the convergence parameters $\varepsilon_{\mathrm{f}}$ and $\varepsilon_{\mathrm{g}}$ are properly sized.

\section{NUMERICAL EXAMPLE}

The design optimization problem of equations (13)-(16) has been solved by using a numerical procedure of Matlab Optimization Toolbox (Grace 1992), that give practical solutions with no great computational efforts in agreement with the flowchart of Figure 11 by assuming $\varepsilon_{\mathrm{f}}=0.001$ and $\varepsilon_{\mathrm{g}}=0.001$.

For the numerical example, the following data have been used from the experimental measures: human finger contact forces as $F_{1}=6.0 \mathrm{~N}, F_{2}=5.5 \mathrm{~N}, F_{3}=6.0 \mathrm{~N}$;

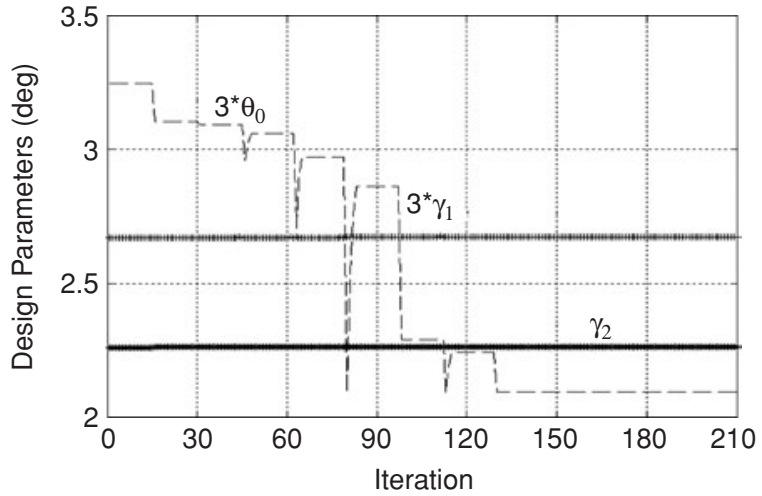

Figure 16 Evolution of the design parameters $\gamma_{2}, \gamma_{1}^{\prime}, \theta_{0}$.

Table 2 Design parameters of the optimal design solution of Figure 12

\begin{tabular}{lcc}
\hline Size $(\mathrm{mm})$ & Initial guess & Optimal solution \\
\hline$l_{53}$ & 23.6 & 26.3 \\
$l_{21}$ & 40.9 & 20.0 \\
$l_{31}$ & 40.5 & 60.0 \\
$l_{41}$ & 5.4 & 2.0 \\
$l_{51}$ & 43.7 & 64.4 \\
$l_{12}$ & 4.8 & 2.0 \\
$l_{22}$ & 26.0 & 27.1 \\
$l_{32}$ & 25.0 & 50.0 \\
$l_{42}$ & 5.4 & 2.2 \\
$l_{52}$ & 22.0 & 22.0 \\
\hline
\end{tabular}

input angle as $\theta_{1}=30^{\circ}$ sizes of human finger phalanx as $L_{\text {phal lh }}=26.9 \mathrm{~mm}, L_{\text {phal } 2 \mathrm{~h}}=21.5 \mathrm{~mm}, L_{\text {phal } 3 \mathrm{~h}}=$ $25.1 \mathrm{~mm}$; human finger diameter as $d_{\text {human }}=18 \mathrm{~mm}$.

The design parameters of the optimal design solution have been obtained after 200 iterations and a computer processing time of $2.41 \mathrm{~s}$ with a Pentium 3 processor. Results are reported in Figures $12-16$ and in Table 2.

\section{CONCLUSIONS}

In this paper we have presented a design procedure for driving mechanisms of anthropomorphic fingers with 1 d.o.f. having suitable characteristics regarding with finger motion, grasping equilibrium and force transmission. The numerical procedure is characterized by fairly simple formulation for the optimality criteria and no great computational efforts in order to achieve practical design solutions. An example has been reported as applied to an enhancement of a 1 d.o.f. anthropomorphic finger mechanism that has been built at LARM in Cassino.

\section{REFERENCES}

Barrett Hand, http://www.barretttechnology.com

Butterfass J, Grebenstein M, Liu H, Hirzinger G. 2001.

DLR-hand II: Next generation of a dexterous robot hand. In: IEEE Interantional Conference on Robotics and Automation, Seoul, Vol. 1, pp. 109-20. 
Carbone G, Civitillo R, Ceccarelli M. 2002. Design and test of an articulated mechanism for a 1-dof anthropomorphic finger. In: ASME Design Engineering Technical Conferences DETC'02, Montreal, paper MECH-34300.

Ceccarelli M. 1997. An optimum design for a gripping mechanism. In: International Symposium Machines and Mechanisms ISMM'97, Beograd, pp. 23-6.

Ceccarelli M, Nava NE, Jauregui JM, Parada JE, Carbone G. 2003a. Diseño y experimentación de un dedo articulado antropomorfo con un grado de libertad. In: VII Congreso Iberoamericano de Ingeniería Mecánica 2003, Coimbra.

Ceccarelli M, Jauregui JM, Parada JE, Nava NE, Lanni C, Carbone G. 2003b. Experimental activity for designing a hand with 1 dof anthropomorphic fingers of human size. In: 12th Interantional Workshop on Robotics in Alpe-Andria-Danube Region RAAD 2003, Cassino, paper 009RAAD03.

Dechev N, Cleghorn WL, Nauman S. 1999. Multiple finger, passive adaptive grasp prosthetic hand. Mechanism Mach Theory, 34(8):1157-73.

Deibe A, Cardenal J, Cuadrado J, Ceccarelli M. 1997. Síntesis optima de mecanismos para pinzas robóticas. In: Spanish National Congress on Mechanical Engineering, Bilbao, pp. 235-42.

Figliolini G, Ceccarelli M. 1999. Epicyclic gearings and timing belts for an articulated finger. In: 4th World Congress on Gearing and Power Transmission, Paris, pp. 2533-8.

Figliolini G, Ceccarelli M. 2000. A mechanical design of one d.o.f. anthropomorphic finger based on a human finger motion. In: ASME Design Engineering Technical Conferences, Baltimore, paper DETC2000/MECH-14132.

Figliolini G, Ceccarelli M. 2002. A novel articulated mechanism mimicking the motion of index finger. Int 7 Robotica, 20:13-22.

Figliolini G, Rea P, Principe M. 2003. Mechatronic design of Ca. U. M. Ha (cassino-underactuated-multifinger-hand). In: 12th International Workshop on Robotics and Alpe-Adria-Danube Region RAAD 2003, Cassino, paper 026RAAD03.

Fukaya N, Toyama S, Asfour T, Dillmann R. 2000. Design of the TUAT/Karlsruhe humanoid hand. In: IEEE/RSJ Internaional Conference on Intelligent Robots and Systems, Takamatsu, pp. 1754-9.
Gosselin CM, Mountambault S, Gosselin CJ. 1993. Manus Colobi: Preliminary results on the design of a mechanical hand for industrial applications. In: 19th ASME Design Automation, Albuquerque, pp. 585-92.

Grace A. 1992. Optimization toolbox user's guide. Natick: The Matlab Works Inc.

Jauregui JM, Parada JE, Nava NE. 2003. Design of an anthropomorphic hand with three 1 dof fingers. Master Thesis, University of Los Andes, Mérida (developed at LARM).

Mason MT, Salisbury JK. 1985. Robots hands and the mechanics of manipulation. Cambridge: MIT Press.

Nava NE, Carbone G, Ceccarelli M. 2004a. Validación experimental de manos robóticas. In: V Congreso Nacional de Ingeniería Mecánica, Mérida, pp. 189-94.

Nava NE, Carbone G, Ottaviano E, Ceccarelli M. 2004b. An experimental validation of a three-fingered hand with 1 dof anthropomorphic fingers. In: Intelligent Manipulation and Grasping 2004, Genova, pp. 285-90.

Penisi HO, Carbone G, Ceccarelli M. 2002. Optimum design and testing of mechanisms for two-finger grippers. In: International Symposium on Multibody Systems and Mechatronics MUSME2002, Mexico City, paper no. M03.

Raparelli T, Mattiazzo G, Mauro S, Velardocchia M. 2000. Design and development of a pneumatic anthropomorphic hand. f Robotic Syst, 17:1-15.

Stanford/JPL Hand, http://www.engineer.tamuk.edu

Taylor GL, Schwartz RJ. 1955. The anatomy and mechanics of the human hand. Artif Limbs, 2:22-35.

Tecnoedu Homepage. Dedo humano de tamaño natural 3B M19, http://www.tecnoedu.com

Townsend WT. 2000. The Barrett Hand grasper-programmable flexible parts handling and assembly. Ind Robot: Int $\mathcal{F}$, 27(3):181-8.

Vanderplaats GN. 1984. Numerical optimization techniques for engineering design. New York: McGraw-Hill.

Zhang Y, Han Z, Zhan H, Shang X, Wang T, Guo W. 2001. Design and control of the BUAA four-fingered hand. In: IEEE Transactions on Robotics and Automation, pp. 2517-22. 

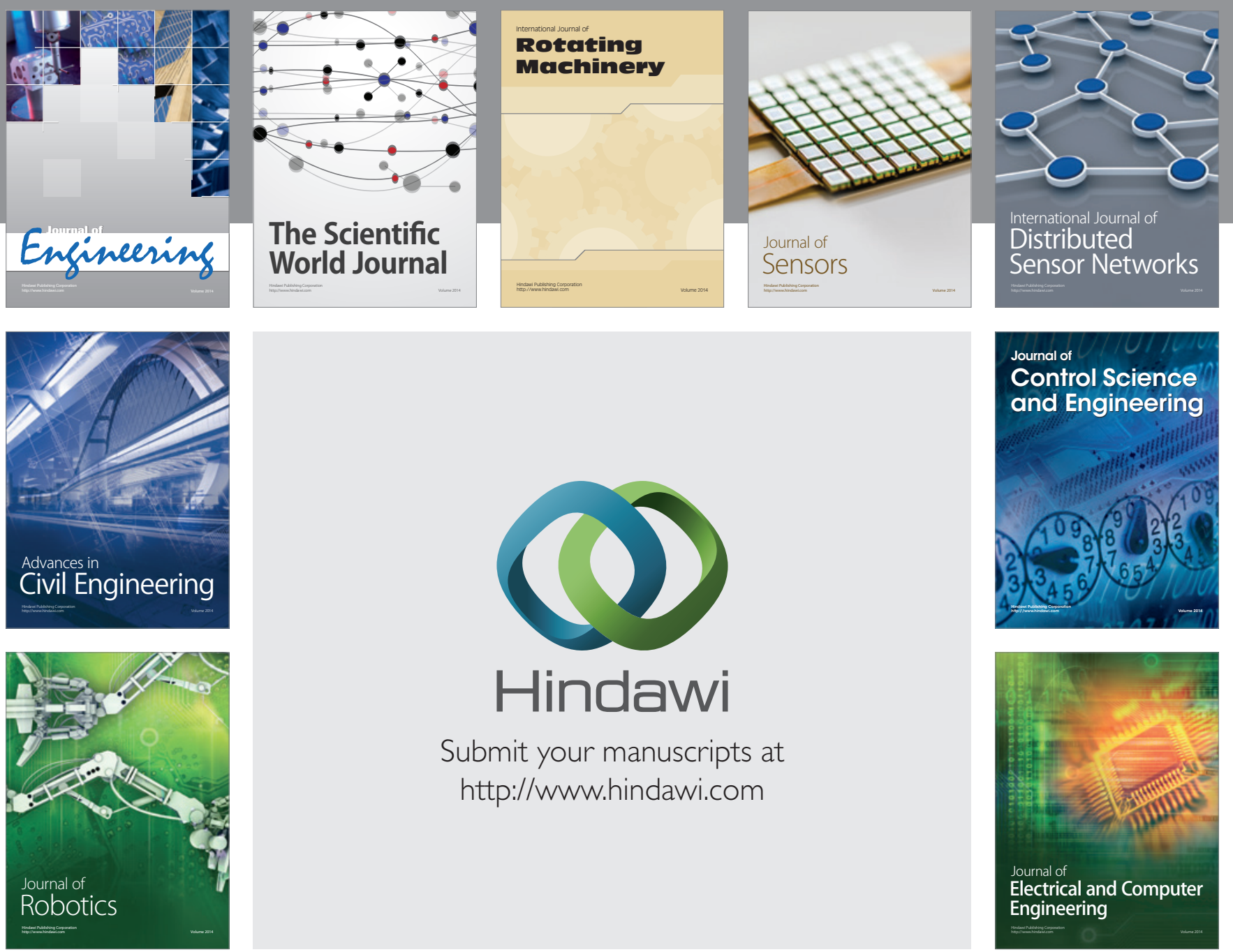

Submit your manuscripts at

http://www.hindawi.com
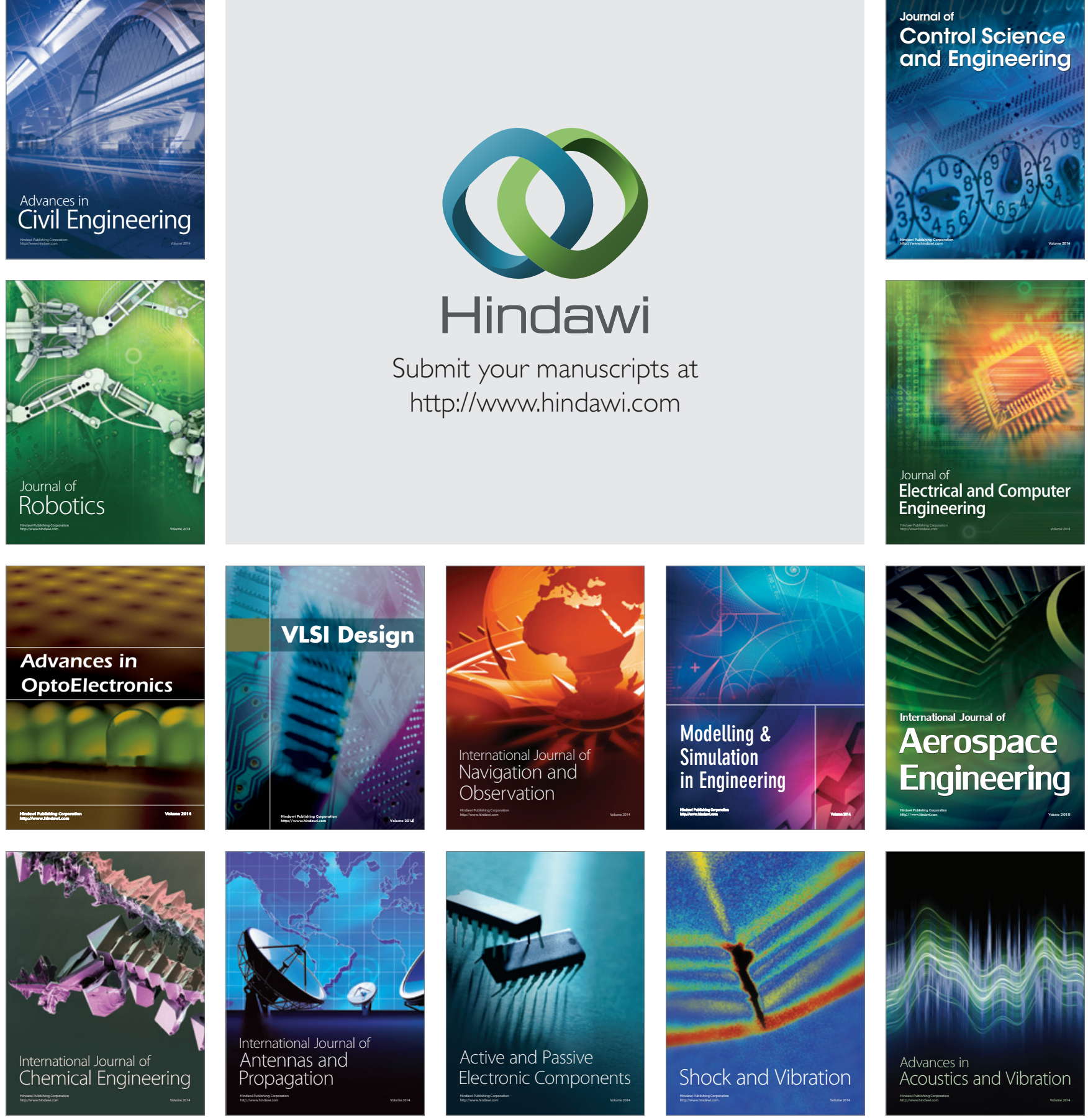